\title{
Heimat und Sprache Überlegungen im Anschluss an Georg Misch
}

\author{
Naemi BREMECKER \\ Philipps-Universität Marburg (Germany)
}

\begin{abstract}
This text seeks to understand the concept of Heimat. I of knowledge and speech that are tied to the individual argue that it is not so much Heimat itself that needs to be examined but rather the subject area which includes experience of the speaker. This insight sheds new light objects such as Heimat. I develop this concept with the on certain confusions of representational references in the discourses on Heimat.

help of Georg Misch's analytical tools. Heimat as an object shows itself in a specific form of speech - „Evozieren" (to evoke). Objects of evoking speech, like objects Keywords: Heimat, Georg Misch, Hermeneutik, Evozierende Rede, practical knowledge, experience
\end{abstract}

of practical knowledge, are subject to specific conditions

(c) Naemi Bremecker; naemibremecker@gmail.com

Colloquium: New Philologies, Volume 6, Issue 1 (2021)

doi: 10.23963/cnp.2021.6.1.10

Stable URL: https://colloquium.aau.at/index.php/Colloquium/article/view/154

This work is licensed under a Creative Commons Attribution 4.0 International License (CC BY 4.0). 
„Jedermann kennt was man mit [...] Heimat meint. Sobald wir uns jedoch aufmachen, dem nachzudenken,[...] wo und wie Heimat waltet, sind wir schon ins Unbestimmte und Bodenlose geraten“, sagt Martin Heidegger $(1983,155)$ in seinem Aufsatz Sprache und Heimat und illustriert so die Denkschwierigkeit, die sich dieser Aufsatz zur Aufgabe gesetzt hat. In der Suche nach einer Antwort auf dieses Problem, wird zunächst mittels einleitender Beobachtungen in den Gegenstandsbereich eingeführt um anschließend, mithilfe einiger theoretischer Überlegungen Georg Mischs, der Antwort auf die Frage nach der Heimat näher zu kommen.

\section{Einleitende Eingrenzung des Problembereichs}

Andrea Bastian versucht in ihrer Dissertation Der Heimat-Begriff. Eine begriffsgeschichtliche Untersuchung in verschiedenen Funktionsbereichen der deutschen Sprache (1995) ein Verständnis von Heimat zu entwickeln. Sie sammelt und systematisiert die Gebrauchsweisen des Wortes Heimat in der deutschen Sprache und kommt zu einem komplexen Ordnungssystem. Sie unterscheidet die Gebrauchsweisen des Wortes schließlich in räumliche, zeitliche, soziale und emotionale kategoriale Zuordnungen für die Funktionsbereiche des Alltags und der Theorie in Recht, Politik, Literatur- und Naturwissenschaften. Auf der letzten Seite ihres Fazits kommt sie schließlich zu dem ernüchternden Schluss: „Es ist von der Existenz eines Heimatbegriffs auszugehen.“ Sein „inhaltliches Spektrum [ist] sehr breit gefächert [...] und [...] schwer einzugrenzen.“ Andrea Bastian folgert: „Es gibt keine allgemeingültige ,eigentliche“ Bedeutung von ,Heimat ““ (Bastian 1995, 221).

Auch wenn Bastian wichtige Beobachtungen macht, scheint das Problem an der Suche nach Heimat darin zu bestehen, dass Heimat fast alles sein kann. Die Dimensionen schwellen an, die Facetten häufen sich und Bastians Funktionsbereiche decken endlich quasi jeden Bereich des Lebens ab. Das Problem besteht letztlich darin, dass Heimat so zwischen ,traditionell gebraut', ,Dresden', oder ,dem Ort der allen in die Kindheit scheint ${ }^{*}$ alles bedeuten kann. Diese Beobachtung ist auf gewisse Weise ernüchternd. Trotzdem kann man etwas aus dem methodischen Vorgehen Bastians lernen.

Folgende drei Eigenschaften von Heimat lassen sich in einer ersten Beobachtung anführen: Heimat ist kein Begriff, der verschiedene Eigenschaften definitorisch enthält. Man kann nur bedingt, wie Bastian es versucht hat, alle Eigenschaften von Heimat, z.B. in den verschiedenen Gebrauchsweisen des Wortes, aufsuchen und sortieren, um dann - aufleitend - zu einer Definition zu kommen. Dies lässt sich nicht nur an dem in seinem Umfang nicht mehr umgrenzten Heimatbegriff Bastians zeigen, sondern auch dadurch, dass man sich folgende Analogie verdeutlicht. Bestimmt man z.B. die Lebensbedingungen einer Pflanzenart, dann untersucht man zunächst deren natürliches Biotop und entdeckt 
bestimmte Eigenschaften. Beispielsweise könnte eine bestimmte Pflanze viel Licht und einen feucht-sumpfigen Moorboden bevorzugen. Es ist dann möglich, diese Eigenschaften an anderen Orten zu finden oder im Gewächshaus zu reproduzieren, und die Pflanze wird an einem anderen Ort mit selben Eigenschaften ebenso gut wachsen können. Heimat kann zwar geographisch-materiell ebenso lokalisierbar sein und dieser Ort kann in seinen Eigenschaften beschreibbar sein, ist aber durch keinen anderen Ort und kein ähnliches Ding ersetzbar. Die Heimat ist ein Einzelnes und Individuelles, welches nicht in der Beschreibung der Summe seiner Teile aufgeht, weil Heimat nicht als ein Ganzes aus Teilen oder eine Summe von Eigenschaften angemessen modelliert werden kann.

Ebenso wie die vorgestellten Methoden nicht funktionieren können, um zu einem Begriff von Heimat zu kommen, ist die gegensätzliche Richtung zwar durchsetzbar, aber problematisch. Entwirft man ein bestimmtes Bild von Heimat und legt die Eigenschaften derselben verbindlich fest, dann führt jede Grenzziehung zum Ausschluss des Anderen. Wird diese Heimat dann auch noch zu einem politisch erwünschten Ziel erklärt, führt dies in seinen abartigsten Formen dazu, dass Menschen davon sprechen können, ,das Reich' müsse vom demjenigen gesäubert werden, was die Verwirklichung von Heimat bedrohen könnte.

Die zweite Beobachtung besteht darin, dass die Eigenschaften, die Heimat zugeschrieben werden, einander widersprechen können und nicht zwingend sind. So kann Heimat beispielsweise für die Außenseiter der Romantik ein unerreichbarer Sehnsuchtsort sein und zugleich das Aufgehen in einer völkischen Gemeinschaft aufdeutschem Boden. Auch individuelle Heimatbegriffe sind unterschiedlich und nicht zwingend. Wenn Menschen beispielsweise aus derselben Region stammen, kann einer von ihnen stolz erklären, eben diese Region sei seine Heimat, während ein anderer behauptet, seine Heimat in Gottes Liebe gefunden zu haben, und ein dritter spricht davon, seine Heimat in den Steppen Afrikas zu haben. Selbst für das einzelne Individuum muss Heimat nicht konsistent sein und man kann erklären, seine Heimat in den Bergen zu finden, um im Laufe der Zeit festzustellen, dass dies nicht mehr gilt und man sich als heimatlos beschreibt.

Der Titel dieses Aufsatzes lautet: Heimat und Sprache. Schon daran, dass zuvor die Wörter „Erklären“ und „Behaupten“ verwendet wurden, kann erahnt werden, dass Sprache und vor allem das Sprechen eine Rolle spielen könnte. Die dritte Beobachtung formuliert sich so zugleich als These: Heimat ist ein Gegenstand, der sich im Sprechen von Sprache vollzieht. Illustrieren wir diesen Gedanken zunächst mit einer einfachen Beobachtung: Es ist ein Unterschied, ob man sagt: „Ich bin in Hamburg geboren“ oder „Ich bin Hamburger. Meine Heimat ist Hamburg “. Der Referent ,Hamburg bleibt in beiden Sätzen bestehen, aber während die erste Sprechweise eine eher nüchterne Beschreibung einer Tatsache ist, wird im zweiten Satz etwas getan. Das Ich des Sprechers, seine indi- 
viduelle Person, wird an den Geburtsort gebunden. Es ist ja mitnichten nötig, den eigenen Geburtsort zur Heimat zu erklären. Man sagt also quasi: ,Ich bin geworden, der ich bin, eben weil ich von genau dort herkommé. Die Sprecherin stellt eine Verbindung zwischen ihrem gegenwärtigen Ich und dem referentiellen Ort der Rede ,Hamburg' her. Damit ist Hamburg aber nicht mehr ein Ort neben anderen Orten, sondern ,Hamburg wird zu demjenigen Ort, den der Sprecher als Ort der Heimat erlebt hat. Wir haben es hier mit einem performativen Akt zu tun, der einen Ort (oder weiter gefasst ein Ding in der Welt) herausstellt und ihn besondert, indem er als bedeutsam für die Sprecherin in einem Akt sprachlicher Anerkennung erklärt wird. Diese sprachliche Bezugnahme, die eine Verortung, Zuordnung und Verknüpfung des Sprechers darstellt, kann für Einzelne sowie Gruppen gelten. Sie ist nicht zwingend, kann ausbleiben, sich ändern oder sich ähneln - aber, und das ist der springende Punkt, sie muss vollzogen werden.

Modifizieren wir also die eingangs gestellte Frage. Wir fragen uns nun nicht mehr, was Heimat ist, sondern was es für Gegenstände sind, die die oben angedeutete Form haben, und kehren damit die Beobachtungsrichtung gewissermaßen um. Es wird nun nicht das Wissen über Heimat untersucht und systematisiert, sondern es sollen Gegenstände eines bestimmten Typs philosophisch entwickelt werden, um in einem zweiten Schritt zu erfragen, ob Heimat ein solcher Gegenstand sein könnte und welcher Mehrwert sich aus einer solchen Betrachtungsperspektive ergeben könnte.

\section{Überlegungen im Anschluss an Georg Misch}

Ich werde die Frage danach, was Heimat für ein Gegenstand ist, mithilfe der philosophischen Begrifflichkeiten Georg Mischs ausschnittsweise analysieren. Misch ist dem sogenannten Göttinger Kreis der 1920-30er-Jahre zuzurechnen und seine ,logische Hermeneutik' siedelt sich inhaltlich zwischen Diltheys Philosophie des Lebens, der Phänomenologie Husserls und Heideggers Fundamentalontologie an. Auch wenn Misch durchaus präsent im philosophischen Diskurs seiner Zeit positioniert war, wird sein Name heute eher mit seinem 4000 Seiten umfassenden Mammutwerk Die Geschichte der Autobiographie verbunden. Vom Reichsbürgergesetz betroffen und zwangsweise in den Ruhestand versetzt, war der den Nationalsozialisten als jüdisch geltende Misch 1935 seines Amtes als Philosophieprofessor in Göttingen enthoben worden und floh schließlich ins englische Exil, ohne seine Lehrtätigkeit zeitlebens wieder aufnehmen zu können (Becker, Dahms \& Wegeler 1998, 295f.). Sein theoretisches Hauptwerk Der Aufbau der Logik auf dem Boden der Philosophie des Lebens. Göttinger Vorlesungen über Logik und Einleitung in die Theorie des Wissens erscheint erst posthum, 1994, nach langjähriger Editionsarbeit. 
Misch ist auf gewisse Weise, nicht nur aufgrund der Repression seiner Werke, ein unzugänglicher Philosoph. Er entwickelt seine Argumente häufig, indem er zwischen zwei Perspektiven wechselt. Dieses methodische Vorgehen ist der Einsicht geschuldet, dass über gewisse Phänomene zwar gesprochen werden kann, sie aber zugleich im Moment des Sprechens und der denkenden Vergegenständlichung modifiziert sind. Der schichtweise Aufbau seiner Logik ist immer von der Unmöglichkeit des Sprechen-Könnens begleitet, vor der Misch nicht schweigt, sondern - immer eingedenk der Verformung - in reflexiv-methodischer Perspektivität oszillierend hin und her geht. Die Weise über Dinge zu reden, die zwar ansprechbar, aber nicht aussagbar sind, nennt Misch die evozierenden Rede.

Im ersten Teil seiner Logikvorlesungen verfolgt er die Entwicklung von einem elementaren Ausdrucksverstehen zu einem diskursiven Sprechen, das Hervorgehen des begrifflichen Denkens aus dem Untergrund eines elementaren Lebensverständnisses, mit einer „erstmaligen Gründlichkeit und Systematik“, so Friedrich Otto Bollnow (1983, 46). Diese Gründlichkeit mit ihren vielfachen Differenzierungen hat allerdings mitunter den Preis der leichten Zugänglichkeit und bedeutet hier, dass wichtige logische und begriffliche Unterscheidungen zwar benannt und skizziert werden, aber es bei Schlaglichtern bleiben muss.

Lautete die These zuvor: Heimat ist ein Gegenstand, der sich im Sprechen von Sprache vollzieht, so lautet sie nun, übersetzt in das Vokabular Mischs: Heimat ist ein hermeneutischer Gegenstand. Ein Wissen um ihn liegt in der Schicht der erlebnishaften Lebensvollzüge und wird in evozierender Rede zum Ausdruck gebracht. Diese These zu verstehen ist Leitsatz der nun folgenden Ausführungen.

Die wichtigste Unterscheidung, die Misch in seinen philosophischen Betrachtungen entwickelt und die gewissermaßen den Schlussstein eines aufwendigen Argumentationsgerüsts bildet, ist die Unterscheidung zweier gänzlich verschiedener Redeformen. Verschieden sind sie in Bezug auf die gegenständliche Beziehung, ihren Wahrheitswert und die Form, in der sie ausgedrückt werden. Begründet liegt dieser Unterschied aber in grundsätzlich verschiedenen Verhaltensweisen des Menschen, Welt sprachlich bedeutsam werden zu lassen. Um die uns interessierende evozierende Seite der Rede zu verstehen, muss zunächst die gegenüberliegende Seite aus Abgrenzungsgründen eingeführt und verstanden werden.

Auf dieser Seite des Spektrums findet sich nämlich die rein-diskursive Rede; eine Rede die wesentlich dadurch gekennzeichnet ist, dass sie sich in Sätzen ausdrückt, die Gegenstände, die so oder so sind und uns im Vorhandensein gegenüberstehen, anspricht und in einem bestimmten Sinn nimmt, in dem Aussagen über diese Dinge getätigt werden (vgl. Misch 1994, 539f.). Ziel einer solchen Weise zu sprechen ist die Eindeutigkeit. In 
den rein-diskursiven Feststellungen ist der Gedanke in den satzmäßigen Formulierungen zum vollen Ausdruck gekommen und der Sachverhalt, auf den sich die Worte meinend beziehen, ist in der Aussage aufgehoben und kann aus ihr entnommen werden. Sofern das Ziel von Sprechen auch immer Verständigung ist und dieselbe am besten gelingt, je eindeutiger die Sprache ist, ist es nicht verwunderlich, dass die rein-diskursiven Aussagen zum Sprachideal, ja bisweilen zur einzig möglichen Weise, Sprache zu denken, erhoben werden.

Am klarsten ist diese Redeform in den Naturwissenschaften ausgeprägt, wo jeder Begriff als Terminus in Definitionen maximale Eindeutigkeit anstrebt und eben diese definitorische Eindeutigkeit in ihrer Allgemeingültigkeit und Indifferenz zu Äußerungssituation, durch geregelte Einführungs- und Inferenzregeln den Erfolg der Wissenschaften sichert (Vgl. Misch 1994, 573). Die Wahrheit der rein-diskursiven Feststellungen betitelt Misch mit "sachlicher Richtigkeit“ und sie besteht in der Wahrheit einzelner Sätze, die sich ihrerseits in der Übereinstimmung des Urteils mit der Sache zeigt. Es wird also über die Dinge geredet und Richtigkeit liegt in der korrekten Anwendung der Worte im Urteil, sodass die sachliche Beschaffenheit des Gegenstandes erfasst wird.

Aber nicht bei allen Gegenständen menschlicher Rede funktioniert eine solche Vorstellung von Sprach-Welt-Relation. Misch erinnert zum Ende seiner Ausführungen an den platonischen Dialog Laches, der ein solches Problem zu umkreisen scheint. Sokrates fragt in diesem Dialog nach der Tapferkeit und er fragt hierbei nicht unbedingt nach der Bedeutung des Wortes, welches man z.B. ermitteln könnte, indem man wie Andrea Bastian die Verwendungsweisen eines Wortes sammelt und systematisiert. Er will also nicht wissen, was die Intention des Wortes, Tapferkeit' je und je sein kann, sondern er will in essentieller Hinsicht wissen, was Tapferkeit ist. Platon verfährt nun in seinen sokratischen Dialogen häufig so, dass er eine Person, die die gesuchte Eigenschaft verkörpert - also tapfer ist - zum Hauptunterredner macht. Misch stellt fest:

Dies ist kein bloßes künstlerisches Mittel der Verlebendigung, sondern eine methodische Notwendigkeit. Denn der, der die betreffende Tugend besitzt, hat ein Wissen von ihr und muss also sagen können, was sie ist [...]. [V]on dem, was wir besitzen, müssen wir ein Wissen haben, wovon wir aber wissen, davon müssen wir auch sagen können, was es ist. (Misch 1994, 575)

Dieser Ausspruch ähnelt dem Problem, dass das Thema dieses Aufsatzes ist. Heidegger hatte gesagt: „Jedermann kennt, was man mit Heimat meint" und auch die Menschen, die von Heimat sprechen, müssten doch ein Wissen von dem haben, von dem sie doch erzählen? Sobald man aber versucht zu sagen, was man weiß und hat, ist man ins Bodenlose geraten, ebenso wie der tapfere Redner bei der Antwort scheitert und sich verwirrt 
und schließlich ausruft: „[...] ich bin ganz unwillig, wie ich, was ich in Gedanken habe, so gar nicht imstande bin zu sagen“" (Platon, Laches 194b).

Woher kommt dieses Versagen? Sokrates hat recht mit seiner Vermutung, dass der Tapfere ein Wissen darum hat, was es heißt tapfer zu sein. Aber dieses Wissen ist verschieden von dem Wissen um rein-diskursive oder theoretische Gegenstände. Man kann nicht auf den Gegenstand zeigen, prädikative Aussagen sammeln, deren definitorischer Identitätspol dann die Tapferkeit ist.

Das Wissen, welches gesucht wird, ist an die individuelle Erfahrung des Tapferen gebunden. Es ist ein Wissen darum, wie es ist, ein Tapferer zu sein und über dieses Wissen lässt sich sprechen. Aber der Tapfere kann es nicht in der - von Sokrates gesuchten Weise tun. Eben an dieser Stelle kommt dann die evozierende Rede ins Spiel. Wir suchen also nicht nur nach der Bedeutung der Worte, nicht nur nach dem, was das Wort ,Heimat bedeutet, sondern danach was Heimat ist. Nach der Bedeutsamkeit, die sich im Vollzug des Lebens und in den individuellen Erfahrungen ergibt. Diese Bedeutsamkeit ist weiter angelegt und, das ist das Spannende bei Misch, ist weder auf Worte, noch Sprache, ja selbst auf eine menschliche Lebensform beschränkt, weshalb im Folgenden noch einmal argumentativ tiefer angesetzt werden muss. Um die Bedeutsamkeit im Sinne Mischs zu verstehen, muss nun zumindest kursorisch in das elementare und das Erlebniswissen eingeführt werden. Heimat, das sei hier voran gestellt, findet sich vornehmlich in eben jener Wissensschicht.

Misch entwickelt im ersten Teil seiner Vorlesungen einen Boden universeller ausdrucksförmiger Bedeutsamkeit und Sinnhaftigkeit, welchen er als elementares Lebensverhalten und Wissen bezeichnet. Elementares Wissen liegt vor der sprachlichen Lebensform des Menschen und kann beispielsweise auch Tieren zugeschrieben werden. Elementares Verstehen ist umwelt- und situationsbezogen. Es ist an leibliche Vollzüge gebunden und unterscheidet noch nicht zwischen Ich und Objekt. Solches Verstehen vollzieht sich unbewusst und rein instinktiv; es bezieht sich schlicht auf leiblich und wortlos erfolgte Vorgänge, die auf elementare Weise etwas bedeuten.

Interessant ist nun, dass Misch davon ausgeht, dass solche vordiskursiven Weisen zu verstehen auch beim Menschen wirksam ist, auch wenn sie in methodischer Hinsicht nicht anders als aus einer bewussten Perspektive zugänglich sind. Ein sehr einsichtiges Beispiel ist die Analyse dessen was passiert, wenn wir uns erschrecken. Erschrickt man, so hat man etwas Begegnendes als bedrohlich verstanden, aber niemand erschrickt, indem er oder sie etwas als etwas Bestimmtes erkennt, es einordnet und anschließend beschließt, dass man erschrecken sollte. Vielmehr hat man etwas verstanden, bedeutet und wahrgenommen. Die Reflexion darüber, was es ist, das uns erschrocken hat, setzt nachträglich ein. 
Zwischen dieses elementare Wissen und einer bewussten Sprachlichkeit schaltet Misch nun in seinem schrittweisen Aufbau die Erlebnisse. Er entnimmt den Erlebnisbegriff dem Gedankengut seines Lehrers Dilthey. Erlebnis, das ist nicht die Bedeutung der Worte, sondern die Bedeutsamkeit der Gegenstände und Dinge für uns. Ein Erlebnis steht uns nicht als Objekt und anderes gegenüber. Es geht aus dem elementaren Wissen insofern hervor, als dass ein Erlebnis für mich noch nicht unterschieden ist von dem, was es für mich ist. Erlebnis meint das, wo man darinnen ist, nicht gegenständlich von ihm wissend, sondern nur unbestimmt wissend um es (Misch 1994, 344). ,Wissen um' ist ein Wissen, welches nur im Vollzug der gemachten Erfahrungen entsteht (Weingarten 2003, 44). Indem ich mich auf Erlebnisse beziehe, beziehe ich mich nicht meinend auf Gegenstände außerhalb meiner selbst, sondern auf mich, der ich den Gegenstand dessen, was ich weiß, zugleich erfahre und so in der Artikulation erfasse. Misch schreibt: „Dem Erlebnis steht nichts gegenüber in freier Ferne, sondern es erfüllt uns, wir besitzen es in Fülle, während das gegenständlich gerichtete, begreifende Denken das eben nicht besitzt, worauf es meinend gerichtet ist" (Misch 1994, 572). Die Erlebnisse, die wir in der Welt machen, lassen ihre Gegenstände bedeutsam sein und zwar auf andere als rein-diskursive Weise; auf eine leibliche Weise, die sich in und aus den individuellen Erfahrungen speist.

Es scheint, als wenn Heimat sich vorwiegend in dieser Schicht des elementaren Erlebniswissens verorten lässt, indem die leiblich-sinnlichen Gefühle vergangener Erlebnisse und Erfahrungen zum Klingen gebracht werden. Schuberts Lindenbaum rauscht von ersehnter Heimatruhe und auch der Heimatminister Horst Seehofer sagt, wenn er 2019 versucht, den Gegenstand seines politischen Tuns in einer Rede zu fassen: „Dort sagt mir jeder Baum eine Geschichte" (Seehofer 2019). Ein weiterer Auslöser für heimatliche Gefühle kann auch der Geruch sein. Christian Graf von Krockow fasst dies zusammen, wenn er sagt: „Heimat riecht nach Harz und nach Heu, nach Kartoffelfeuern, Leder, Kuchenbacken, gebrannten Mandeln, [...] Oder sei's Schweinemist, Ruß und Rauch: sie riecht jedenfalls“ (Krockow 1984). Wir hatten diese Beobachtung auch schon zuvor angesprochen, als wir den Geburtsort Hamburg von der Heimat unterschieden haben. Das heimatliche Hamburg, welches zu solchem erklärt wird, weil wir von dort kommen und dort ein persönlicher Erfahrungs- und Erlebnissschatz den zunächst nüchternen Ort zum Ort für uns macht. Ein Ort, der durch die Erlebnisse die wir in ihm gemacht haben, zur Heimat und auf elementar-leibliche Weise mit unserem jetzigen Ich verknüpft wird.

An dieser Stelle stellt sich die Frage, wo eigentlich das Problem liegt? Warum wird Heimat zum utopischen Sehnsuchtsort erklärt und wir gehen nicht einfach zurück zur heimatlichen Scholle? Es lassen sich zwei Probleme anführen:

(a) Sprechen wir von Erlebnissen, so müssen wir über die konstitutionellen Eigenschaften dessen nachdenken, was wir Erlebnis nennen. Hans-Georg Gadamer arbeitet 
treffend heraus, dass jedes Erlebnis aus der Kontinuität des Lebens herausgehoben ist und zugleich auf das Ganze des Lebens bezogen ist (vgl. Gadamer 1960, 75). Er schreibt: „Wenn etwas ein Erlebnis genannt oder als Erlebnis gewertet wird, so ist es durch seine Bedeutung zur Einheit eines Sinnganzen zusammengeschlossen." (Gadamer 1960, 729) Diejenigen, die etwas aus dem Fluss des Lebens heraussondern, indem sie ihm Bedeutung auf beispielsweise seine Heimatlichkeit zusprechen, sind selbst die Träger der erinnerten Erlebnisse, aber wenn sie das Erlebnis auf diese Weise herausstellen, so ist es notwendig vergangen. „Was Erlebnis genannt werden kann, konstituiert sich in der Erinnerung“, stellt Gadamer $(1969,72)$ fest, und auch Bollnow formuliert: „Die Bedeutung, die ein Ereignis in unserem Leben hat, ist nicht im Augenblick des Erlebens selbst zu erfassen, sondern ergibt sich erst nachträglich in dem rückwärts in die Vergangenheit gerichteten Blick der Erinnerung." (Bollnow 1980, 10) Erkennen wir etwas Erfahrenes als Heimat, so ist es vergangen.

(b) Wie erinnern wir uns? Die Form, in der Erlebnisse aus dem Fluss mannigfaltiger Lebensvollzüge zu eigener Gegenständlichkeit werden, muss nun thematisiert werden. Erlebnisse und erst recht das elementare Wissen sind nicht unmittelbar verfügbar. ${ }^{1}$ Sie sind ja gerade durch ihr unbewusstes Darinsein gekennzeichnet. Misch hatte seinen Aufbau der Logik mit der Feststellung begonnen, dass gewisse Ausdrucks-, Bedeutungs- und Sinnformen universell sind und sich in vordiskursiven Wissensformen finden und von dort bis in die menschliche Rede ziehen. Die evozierende Rede ist strukturell analog zu der Form des Erlebniswissens, insofern auch die evozierende Rede erfahrungsgebunden und vollzugsgebunden ist. Aber nicht nur formell sind diese beiden Formen des Wissens analog. Sie sind aufeinander bezogen, da das elementare Leibeswissen und das erfahrungsgebundene Erlebniswissen den Gegenstand stellen, der in evozierender Rede ausgedrückt wird. Wir hatten anfangs festgestellt, dass Misch zwischen einer schrittweise aufbauenden Perspektive und einer solchen Perspektive hin und her geht, die die sprachliche Artikulation an den methodischen Anfang stellt. Die Gegenstände des Elementaren und leiblich Erfahrenen sind gewissermaßen unverfügbar und können erst in sprachlicher Artikulation objektiviert, d.h. zu Gegenständen werden, auf die man sich beziehen kann. Damit ist zwar davon auszugehen, dass sie auf gewisse Weise vorsprachlich zuhanden sind, aber zugleich sind sie erst nachträglich überhaupt fassbar. Das Erlebnis steht uns nicht als Anderes gegenüber, sondern es erfüllt uns. Wir besitzen es bereits in Fülle und wissen von dem, was wir haben, indem das Wissen sich reflexiv auf sich selbst rückwendet. Diese vergegenständliche Objektivierung der erfahrenen Erlebnisse vollzieht sich im Medium der Sprache. Hier stellt sich die Macht des Wortes ein, und zwar so, dass durch die

\footnotetext{
1 Misch fasst dies bündig, wenn er gegen Dilthey einwendet: „[W]ir bewegen uns in einer Ausdruckswelt und nicht in einer Erlebniswelt." (Misch 1994, 78)
} 
Kraft des treffenden Ausdrucks die Bewusstwerdung des Erlebnisses erfolgt (Misch 1994, 512f.). Es handelt sich um einen ursprünglichen primären Zugang zu den uns umweltlich begegnenden Dingen, einem so etwas, das uns begegnet und welches sich im treffenden Ausdruck der Evokation einfangen lässt, bewusst wird, gegenständlich wird, vermitteln lässt und so objektiviert.

Ein evozierender Ausdruck ist nicht an die klassische Satzform gebunden, sondern kann auch größeren Umfang haben, ein Gedicht oder das Ganze eines Romans betreffen, oder sich in einem einzigen treffenden Wort erschöpfen. Auch wenn etwas immer in Sprache ausgedrückt ist, ist der Gegenstand des Sprechens nicht aus einer korrekten Sprachanwendung ableitbar, sondern geht von dem ausgedrückten Gedanken selbst aus, der in einem intuitiven, sprachlichen Erfassen produktiv den treffenden Ausdruck findet, um eben jenes individuelle Gefühl des Wissens darum, wie es ist, etwas lebensweltlich zu erfahren, zu treffen. Die Dynamik der evozierenden Ausdrücke unterscheidet sich von den rein-diskursiven Aussagen dadurch, dass sie nur durch den Vollzug der in ihnen ausgedrückten Bewegung fassbar sind und immer neu vergegenwärtigt und vollzogen werden müssen, um verstanden zu werden (Misch 1994, 37). Hermeneutische Gegenstände sind so an den Vollzug der einzelnen Rede und die in ihr zur Sprache kommenden Gegenstände gebunden. Erst im Zur-Sprache-kommen selbst entstehen die Gegenstände als solche. Misch stellt fest, dass „[w]ährend für die Naturwissenschaften also noch galt, dass Naturwissenschaftliche Begriffe, einmal gewonnen, bestehen und als feste Größe genommen werden können“, evozierende Ausdrücke, gerade an Ausdruckskraft verlieren, wenn sie in terminologischen Gebrauch genommen werden, und „dieses schnelle Sichverbrauchen der Ausdruckskraft eines ursprünglich treffenden Wortes kommt aber daher, weil die Ausdruckskraft an den Vollzug des darin ausgedrückten Wissens gebunden ist“" (Misch 1994, 551).

Auch Heimat ist ebenso eine hermeneutische Gestaltung, die im Vollzug der evozierenden Rede zu ihrem Ausdruck kommt. Wir haben es hier mit keinem Randphänomen der Sprache zu tun, sondern mit einer umfassenden Weise des Menschen, sich selbst zu verstehen. Auch Misch ist eine Untersuchung der spezifischen Form der Versprachlichung der Erlebnisse und deren Bedeutung ein spezielles Anliegen. Das zeigt sich schon daran, dass er einen großen Teil seiner Produktivität darauf verwendet hat, die Resultate eben solcher Selbstäußerungen zu untersuchen. Er versteht Autobiographie als eine Äußerung des Wissens des Menschen von sich selbst (vgl. Misch 1949, 10).

Die menschliche Lebensform unterscheidet sich von allen anderen Lebensformen dadurch, dass sie grundlegend sprachlich strukturiert ist. Mit dem gemeinschaftlichen Vollzug des Sprechens von Sprache - das entwickelt Misch eindringlich im Aufbau der Logik, ohne dass es hier en détail ausgeführt werden kann - entsteht die Möglichkeit von Selbst- 
wie Gegenstandsbewusstsein. Es ist also nicht nur so, dass wir die Möglichkeit haben, einen wie auch immer gearteten mentalen Zustand des Gefühls oder der Wahrnehmung in Sprache auszudrücken und auf die Welt zu beziehen, sondern vielmehr vorhergehend so, dass eben jene Welt in einem ursprünglich zu denkenden Akt sich selbst im Wort objektiviert und so mentale Zustände als bewusste Zustände allererst ermöglicht. Das bedeutet aber auch, dass wir keinen anderen als einen sprachlichen Zugriff auf unsere Erlebnisse haben.

Mischs Schüler Josef König formuliert das in dem Aufsatz Georg Misch als Philosoph:

[D]as primäre Wissen vom Erwirken und von den Erlebnissen überhaupt ist für uns nur in der Gestalt eines im Spiegel des Ausdruck Erblickten da.

Daß die Vergegenständlichung die Erlebnisse in einem gewissen Sinne hervorbringt, korrespondiert dem, daß es Erlebnisse nur in der Gestalt von Zurückgespiegelten gibt. (König 1967, 231)

Das ursprüngliche Eingebundensein und Erleben sind damit in ihrer Unmittelbarkeit nicht mehr zu erreichen. Gadamer formuliert diesen Gedanken: „Erhebung über die Umwelt dagegen ist für den Menschen Erhebung zur Welt und bedeutet nicht verlassen der Umwelt, sondern eine andere Stellung zu ihr, ein freies, distanziertes Verhalten, dessen Vollzug jeweils ein sprachlicher ist." (Gadamer 1960, 448f) Die bewusste Freiheit schafft aber zugleich eine Distanz. Wir versuchen sprechend in einem „zweiten Gang“ in der Redeform des Evozierens die Unmittelbarkeit wieder herzustellen, indem wir uns erzählen, erzählen wie es ist, zu sein. Und da wir eine Lebensform und eine Sprache teilen, so kann es passieren, dass ein Ausdruck trifft und verstanden wird, weil er eine grundlegende Erfahrung evoziert.

\section{Fazit}

Vielleicht ist Heimat ein solcher Ausdruck, der eben jene Unmöglichkeit der Rückkehr in einen kindlich-utopischen Zustand der Selbstverständlichkeit nostalgisch trifft. Nostalgisch, weil wir an der Unmöglichkeit der Rückkehr leiden, denn auch wenn die Orte der gemachten Erfahrungen bestehen bleiben mögen, so sind die Lebensvollzüge in ihnen längst vergangen, und nicht mehr erreichbar, oder eher nie anders als sprachlich distanziert zu erreichen. Spricht der Mensch über Heimat, dann ist anzunehmen, dass sie nicht mehr selbstverständlich ist. Dann befindet sich der Mensch im Exil, sieht seine Lebensweise von Fremden oder Anderem bedroht, oder wendet sich gegen eine fortschreitende Modernisierung, Technisierung oder Globalisierung, die ihn vom locus amoenus seiner selbstverständlichen Zugehörigkeit entfernt. Am Anfang war Heimat heißt ein 2016 erschienenes Buch von Eberhard Rathgeb und der Autor hat recht und unrecht mit dem 
Titel. Heimat muss anfänglich gedacht werden, da sich die Erzählung von Heimat immer auf ein zuvor Gewesenes bezieht, doch zugleich kann Heimat als solche überhaupt erst im Akt der Versprachlichung, im Vollzug ihres Sprechens gedacht werden und entsteht erst, wenn ein Ausdruck es schafft, dass Gefühl des Erlebten zu fassen. Am methodischen Anfang steht das Wort. ${ }^{2}$

Eine Beschäftigung mit Heimat, die diese begrifflichen Eigenschaften nicht bedenkt, ist gefährlich. Denkt man Heimat als real zu errichtenden Ort mit klar umgrenzbaren Eigenschaften, dann wird Heimat problematisch. Ein solcher unreflektierter Heimatforderer versucht, Heimat zu fixieren, ohne zu verstehen, dass ein jeder seine Heimat selbst erzählen muss, weil jede Erfahrung als solche individuell ist. Ein solcher Heimatforderer vergisst, dass Heimat zugeschrieben werden kann und erst recht nicht an nationalen Grenzen und Abstammung fixiert sein muss, sondern, dass man in einem Akt der Anerkennung seine heimatliche Verortung selbst vollziehen muss. Er bemerkt nicht, dass Heimat in dem Moment, in dem er beginnt sie zu fordern, nicht mehr selbstverständlich und einfach erreichbar ist.

Und trotzdem dürfen wir nicht aufhören, über Heimat zu sprechen. Heimat vollzieht sich doch gerade im Erzählen von ihr; eine Erzählung von uns selbst, die davon erzählt, wer wir sind, indem wir erzählen, woher wir kommen. In der Rede von Heimat kann man finden, woher man kommt, woran man krankt, was fehlt, und wohin man will. Beheimatung ist ein identitätsbildender Akt von Individuen wie Gruppen, eine geistige Bewegung, die ihr Ziel in sich selber trägt und die sich im sprechenden Vollzug immer wieder neu hervorbringen muss. Ein in diesem Sinne aufgeklärtes Sprechen über Heimat ist wichtig, wenn das Selbstverständnis angesichts immer weniger Selbstverständlichkeiten neu stabilisiert werden muss.

\section{Literatur}

Bastian, Andrea. 1995. Der Heimat-Begriff. Eine begriffsgeschichtliche Untersuchung in verschiedenen Funktionsbereichen der deutschen Sprache. Berlin: De Gruyter.

\footnotetext{
2 Maja Soboleva kommt in ihrem jüngst erschienenen Aufsatz Ist, Heimat' ein Mythos? Der Heimatbegriff zwischen Bezeichnung und Bedeutung (2020) zu dem Schluss, dass Heimat atopisch und achronisch verstanden werden könne (vgl. Soboleva 2020, 520). Da eine örtliche Bindung nicht notwendig sei und Heimat „nur im Moment des Sprechens (Denkens und Vorstellens) vorhanden ist“ (ibid., 522), deutet sie Heimat letztlich als Mythos, der lediglich "reine" Bedeutung und „ideellen Charakter" enthalte (ibid., 527). Hier wird hingegen behauptet, das Sprechen über Heimat stehe am methodischen Anfang, verweise aber zugleich auf ein zuvor Erlebtes, welches durchaus örtlich gebunden sein kann. Heimat ist nicht konstruktivistisch zu denken, sondern die Aufgabe besteht vielmehr darin, die Verweisstruktur der Heimatrede ernst zu nehmen, aber zugleich als Akt sprachlicher Anerkennung zu verstehen. Damit hat die Rede über Heimat die eigenwillige zeitliche Struktur einer gleichzeitigen Vorzeitigkeit.
} 
Bollnow, Otto Friedrich. 1980. „Lebensphilosophie und Logik. Georg Misch und der Göttinger Kreis“, Zeitschrift für philosophische Forschung, Bd. 34, Heft 3, 423-440.

Bollnow, Friedrich Otto. 1983. Studien zur Hermeneutik. Band II: Zur hermeneutischen Logik von Georg Misch und Hans Lipps. Freiburg und München: Alber Verlag.

Gadamer, Hans-Georg. 1960. Wahrheit und Methode. Grundzüge einer philosophischen Hermeneutik. Tübingen: J. C. B. Mohr (Paul Siebeck).

Heidegger, Martin. 1983. „Sprache und Heimat“. In Martin Heidegger: Aus der Erfahrung des Denkens 1910-1976, Bd. 13, 155-180. Frankfurt am Main: Vittorio Klostermann.

Heinrich Becker, Hans-Joachim Dahms, Cornelia Wegeler (Hg.). 1998. Die Universität Göttingen unter dem Nationalsozialismus. München: K. G. Saur.

König, Josef. 1967. „Georg Misch als Philosoph“, Nachrichten der Akademie der Wissenschaften in Göttingen, Nr. 7, 152-243.

Krockow, Christian Graf von. „Heimat.“ Die Zeit, 05.10.1984.

Misch, Georg. 1949. Geschichte der Autobiographie, Bd. 1. Frankfurt am Main: Schulte-Bulmke.

Misch, Georg. 1994. Der Aufbau der Logik auf dem Boden der Philosophie des Lebens. Göttinger Vorlesungen über Logik und Einleitung in die Theorie des Wissens. München: Alber Verlag.

Platon. 1977. Laches. In Platon. Werke in acht Bänden. Griech. u. dt., Bd. 1, herausgegeben von Gunter Eigler. Darmstadt: Wissenschaftliche Buchgesellschaft.

Rathgeb, Eberhard. 2016. Am Anfang war Heimat. Auf den Spuren eines deutschen Gefühls. München: Karl Blessing.

Weingarten, Michael. 2003. „Leben (bio-ethisch).“ In Bibliothek dialektischer Grundbegriffe, Bd. 2, herausgegeben von Andreas Hüllinghorst. Bielefeld: transcript.

Seehofer, Horst. „Dort sagt mir jeder Baum eine Geschichte. Horst Seehofer im Gespräch mit Tina Hildebrand und Andreas Lebert." Die Zeit, 16.03.2019.

Soboleva, Maja. 2020. „Ist „Heimat‘ ein Mythos? Der Heimatbegriff zwischen Bezeichnung und Bedeutung “. Deutsche Zeitschrift für Philosophie: Zweimonatsschrift der internationalen philosophischen Forschung, 68(4), 514-531. 